\title{
Minimal Weighted Clones with Boolean Support
}

\author{
Peter G. Jeavons, Andrius Vaicenavičius and Stanislav Živný \\ Department of Computer Science, University of Oxford, UK \\ \{peter.jeavons, andrius.vaicenavicius, standa.zivny\}@cs.ox.ac.uk
}

\begin{abstract}
We study algebraic structures called weighted clones. These structures characterise the computational complexity of discrete optimisation problems of special form, known as valued constraint satisfaction problems. We identify all minimal weighted clones for every Boolean support clone.
\end{abstract}

\section{INTRODUCTION}

The Valued Constraint Satisfaction Problem (VCSP) is a general framework of discrete optimisation problems [7], [26]. It is a generalisation of the Constraint Satisfaction Problem (CSP), a framework that captures fundamental decision problems from artificial intelligence, database theory, logic, and graph theory [9]-[11], [17]. The VCSP framework, in addition to the problems above, captures many discrete optimisation problems from combinatorial optimisation and computer vision [7], [26].

In analysing the computational complexity of the CSP with restricted constraint types (the so-called non-uniform CSP), the algebraic approach was one of the main tools [1] [3], [5], [13]. This approach was obtained by estalishing the link between CSPs and universal algebra in [4], [15], [16]. Recently, the link between VCSPs and the algebraic structures, called weighted clones, has also been established [6]. This algebraic approach has been used to classify the complexity of various fragments of the VCSP [12], [19], [22] and to reduce the question of whether the complexity of the VCSP has a dichotomy to the well-established Algebraic CSP Dichotomy Conjecture [18], [20].

Weighted clones are known to capture the computational complexity of the VCSP with any specified set of valued constraints [6]. In particular, weighted clones with Boolean support clone capture the complexity of the VCSP on the Boolean domain. In this paper we classify all minimal weighted clones for each possible Boolean support clone. These minimal weighted clones correspond to maximal weighted relational clones and hence to maximal sets of possible valued constraints. We refer the reader to the recent survey [14] that provides motivation for this work and connects it to the computational complexity of the VCSP. Other recent work on weighted clones includes [23]-[25].

\section{PRELIMINARIES}

Let $D$ be finite a set. A function $f: D^{k} \rightarrow D$ is called a $k$-ary operation on $D$. We denote by $\mathbf{O}_{D}$ the set of all finitary operations on $D$ and by $\mathbf{O}_{D}^{(k)}$ the $k$-ary operations in $\mathbf{O}_{D}$.

A.V. is supported by an EPSRC Doctoral Training Award. S. Ž. is supported by a Royal Society University Research Fellowship.
Definition II.1. The $k$-ary projections on $D$ are the operations $\pi_{i}^{(k)}: D^{k} \rightarrow D$ such that $\pi_{i}^{(k)}:\left(x_{1}, \ldots, x_{k}\right) \mapsto x_{i}$, where $i \in\{1, \ldots, k\}$ and $\left(x_{1}, \ldots, x_{k}\right) \in D^{k}$.

Definition II.2. Let $f \in \mathbf{O}_{D}^{(k)}$ and $g_{1}, \ldots, g_{k} \in \mathbf{O}_{D}^{(\ell)}$. Define the superposition of $f$ and $g_{1}, \ldots, g_{k}$ to be the $\ell$-ary operation $f\left[g_{1}, \ldots, g_{k}\right]: D^{\ell} \rightarrow D$ such that for any $\left(x_{1}, \ldots, x_{\ell}\right) \in D^{\ell}$

$$
\left(x_{1}, \ldots, x_{\ell}\right) \mapsto f\left(g_{1}\left(x_{1}, \ldots, x_{\ell}\right), \ldots, g_{k}\left(x_{1}, \ldots, x_{\ell}\right)\right) .
$$

Definition II.3. A set $C \subseteq \mathbf{O}_{D}$ is called a clone of operations on $D$ if it contains all the projections on $D$ and is closed under superposition. For each $F \subseteq \mathbf{O}_{D}$ we define $\operatorname{Clone}(F)$ to be the smallest clone containing $F$. We denote by $C^{(k)}$ the $k$-ary operations in clone $C$.

Example 1. For any set $D$, the set $\mathbf{J}_{D}$ containing all projections on $D$ and the set $\mathbf{O}_{D}$ containing all operations on $D$ are clones.

Example 2. For any totally ordered set $D$, the clone $C:=$ Clone $(\{\wedge\})$, where $\wedge$ is the binary minimum operation on $D$, consists of all operations on $D$ which return the minimum element of some (non-empty) subset of their arguments. In particular, $C^{(2)}$, the set of binary operations in $C$, contains precisely three operations, namely, $\pi_{1}^{(2)}, \pi_{2}^{(2)}$, and $\wedge$.

Definition II.4. We define a $k$-ary weighting of a clone $C$ to be a function $\omega: C^{(k)} \rightarrow \mathbb{Q}$ such that $\sum_{f \in C^{(k)}} \omega(f)=0$, and $\omega(f)<0$ only if $f$ is a projection. We denote by $\mathbf{W}_{C}^{(k)}$ the set of all $k$-ary weightings of $C$, and by $\mathbf{W}_{C}$ the set of all possible weightings of $C$ (of arbitrary arity).

For $\omega: C^{(k)} \rightarrow \mathbb{Q}$, we write $\left(\alpha_{1}, \ldots, \alpha_{m} \mid f_{1}, \ldots, f_{m}\right)$, where $\alpha_{i} \in \mathbb{Q}$ and $f_{i} \in C^{(k)}$, if for every $f \in C^{(k)}$

$$
\omega(f)=\sum_{\substack{i \in\{1, \ldots, m\} \\ f_{i}=f}} \alpha_{i} .
$$

Note that some of $f_{i}$ might be equal.

Example 3. The following functions are weightings of Clone $(\{\wedge\})$, which was described in Example 2:

$$
\left(-1,1 \mid \pi_{1}^{(2)}, \wedge\right) \text { and }\left(-2,1,1 \mid \pi_{1}^{(2)}, \pi_{2}^{(2)}, \wedge\right) \text {. }
$$

The following functions are not weightings:

$$
\left(1,-1 \mid \pi_{1}^{(2)}, \wedge\right) \text { and }\left(-1,-1,3 \mid \pi_{1}^{(2)}, \pi_{2}^{(2)}, \wedge\right)
$$

as the first assigns a negative weight to a non-projection and in the second the weights do not add up to zero. 
Definition II.5. For any $\omega=\left(\alpha_{1}, \ldots, \alpha_{m} \mid f_{1}, \ldots, f_{m}\right) \in$ $\mathbf{W}_{C}^{(k)}$ and any $g_{1}, \ldots, g_{k} \in C^{(\ell)}$, we define the superposition of $\omega$ and $g_{1}, \ldots, g_{k}$ to be the function $\omega\left[g_{1}, \ldots, g_{k}\right]: C^{(\ell)} \rightarrow \mathbb{Q}$ given by $\left(\alpha_{1}, \ldots, \alpha_{m} \mid f_{1}\left[g_{1}, \ldots, g_{k}\right], \ldots, f_{m}\left[g_{1}, \ldots, g_{k}\right]\right)$.

If the result of the superposition is a valid weighting, then it is said to be a proper superposition.

Definition II.6. A weighted clone, $W$, is a non-empty set of weightings of some fixed clone $C$ which is closed under:

(i) nonnegative scaling by rational numbers;

(ii) addition of weightings of equal arity; and

(iii) proper superposition with operations from $C$.

The clone $C$ is called the support of $W$. For any $S \subseteq \mathbf{W}_{C}$, we define $\langle S\rangle$ to be the smallest weighted clone containing $S$ and say that $\langle S\rangle$ is generated by $S$. We write $\left\langle\omega_{1}, \ldots, \omega_{n}\right\rangle$ for the weighted clone $\left\langle\left\{\omega_{1}, \ldots, \omega_{n}\right\}\right\rangle$.

For a weighting $\omega \in \mathbf{W}_{C}^{(k)}$, we say that $\omega$ is a zero-valued weighting if $\omega(f)=0$ for all $f \in C^{(k)}$. Otherwise, we say that $\omega$ is a non-zero weighting. A weighted clone that contains a non-zero weighting is said to be non-trivial.

Example 4. For any clone $C$, the set $\mathbf{W}_{C}^{0}$ containing all zero-valued weightings of $C$, and the set $\mathbf{W}_{C}$ containing all possible weightings of $C$, are both weighted clones with support $C$.

For any two weighted clones $W^{\prime}$ and $W^{\prime \prime}$ with support $C$, we define $W^{\prime} \wedge W^{\prime \prime}:=W^{\prime} \cap W^{\prime \prime}$ and $W^{\prime} \vee W^{\prime \prime}:=$ $\left\langle W^{\prime} \cup W^{\prime \prime}\right\rangle$. For any clone $C$ on a finite set $D$, the set of all weighted clones with support $C$ ordered by inclusion forms a complete lattice, with the meet and join operations as defined above, and with the least element $\mathbf{W}_{C}^{0}$ and the greatest element $\mathbf{W}_{C}$. We denote this lattice by Lat $(C)$ and call it the lattice of a clone $C$.

Recall that an atom is a minimal non-trivial element in a lattice. A minimal weighted clone is a weighted clone $W$ such that for any non-zero weighting $\omega \in W$ we have $\langle\omega\rangle=W$. Note that a weighted clone $W$ is minimal if, and only if, it is an atom of Lat $(C)$, where $C$ is the support of $W$.

We will use the following two results. The first gives a uniform way to obtain any weighting in a weighted clone.

Lemma II.7 ( [25, Corollary 3.13], [24, Corollary 2.2.4]). Let $C$ be a clone and $S \subseteq \mathbf{W}_{C}$ be a set of weightings. Let $W$ be the smallest weighted clone containing $S$, that is, $W=\langle S\rangle$. Then for each $k$-ary weighting $\omega \in W$ there are weightings $\omega_{1}, \ldots, \omega_{n} \in S$ of arities $k_{1}, \ldots, k_{n}$ respectively, scalars $c_{1}, \ldots, c_{n} \in \mathbb{Q}_{\geq 0}$, and $k$-ary operations $g_{1}^{1}, \ldots, g_{k_{n}}^{n} \in C^{(k)}$, such that

$$
\omega=\sum_{i=1}^{n} c_{i} \cdot \omega_{i}\left[g_{1}^{i}, \ldots, g_{k_{i}}^{i}\right]
$$

Note that some of the $\omega_{i}$ can be equal and improper superposition is allowed in (2).

The second result gives a sufficient condition for a weighting to generate the weighted clone of all weightings, $\mathbf{W}_{C}$.
Lemma II.8 ( [25, Theorem 3.2]). Let $\omega$ be a non-zero weighting of a clone $C$ that assigns positive weight to some projection. Then $\omega$ generates the weighted clone containing all weightings of $C$, that is, $\langle\omega\rangle=\mathbf{W}_{C}$.

\section{MAIN RESUlts}

In this paper we focus on Boolean clones, consisting of operations defined on the domain $B:=\{0,1\}$, with the usual order $0<1$. We will use the following six standard Boolean operations: $\mathbf{0}, \mathbf{1}, \neg, \wedge, \vee, \mathrm{mjr}$, mnr, which we call key operations. They are defined, respectively, to be the unary constant 0 operation, the unary constant 1 operation, the unary inverse operation given by $x \mapsto 1-x$, the binary operation returning the smaller of its two arguments, the binary operation returning the larger of its two arguments, the unique ternary operation on $B$ such that $\operatorname{mjr}(x, x, y)=\operatorname{mjr}(x, y, x)=$ $\operatorname{mjr}(y, x, x)=x$ for any $x, y \in B$ (known as the majority operation), and the unique ternary operation on $D$ such that $\operatorname{mnr}(x, x, y)=\operatorname{mnr}(x, y, x)=\operatorname{mnr}(y, x, x)=y$ for any $x, y \in B$ (known as the minority operation).

The next result identifies a special set of weightings, called key weightings and shows that every non-trivial weighted Boolean clone must contain a key weighting. This implies that every minimal weighted clone is generated by a key weighting. Since there are only nine key weightings, for any Boolean clone $C$, Lat $(C)$ has at most nine candidates for atoms.

Theorem III.1 ( [6, Theorem 8.1]). Let $C \neq \mathbf{J}_{B}$ be a clone on $B$. Then any non-trivial weighted clone $W$ with support $C$ must contain at least one of the following weightings:

1) $\left(-1,1 \mid \pi_{1}^{(1)}, f\right)$, where $f=\mathbf{0}, \mathbf{1}$ or $\neg$;

2) $\left(-1,-1,2 \mid \pi_{1}^{(2)}, \pi_{2}^{(2)}, f\right)$, where $f=\wedge$ or $\vee$, or $\left(-1,-1,1,1 \mid \pi_{1}^{(2)}, \pi_{2}^{(2)}, \wedge, \vee\right)$

3) $\left(-1,-1,-1,3 \mid \pi_{1}^{(3)}, \pi_{2}^{(3)}, \pi_{3}^{(3)}, f\right)$, where $f=\operatorname{mjr}$ or mnr, or

$$
\left(-1,-1,-1,2,1 \mid \pi_{1}^{(3)}, \pi_{2}^{(3)}, \pi_{3}^{(3)}, \mathrm{mjr}, \mathrm{mnr}\right) \text {. }
$$

Our notations for these key weightings, and the weighted clones they generate, are listed in Table I. We add a subscript

TABLE I

\begin{tabular}{|c|c|}
\hline Weighted clone & Generating key weighting \\
\hline$W^{\mathbf{0}}$ & $\omega^{\mathbf{0}}:=\left(-1,1 \mid \pi_{1}^{(1)}, \mathbf{0}\right)$ \\
\hline$W^{1}$ & $\omega^{\mathbf{1}}:=\left(-1,1 \mid \pi_{1}^{(1)}, \mathbf{1}\right)$ \\
\hline$W^{\urcorner}$ & $\omega\urcorner:=\left(-1,1 \mid \pi_{1}^{(1)}, \neg\right)$ \\
\hline$W^{\wedge}$ & $\omega^{\wedge}:=\left(-1,-1,2 \mid \pi_{1}^{(2)}, \pi_{2}^{(2)}, \wedge\right)$ \\
\hline$W^{\vee}$ & $\omega^{\vee}:=\left(-1,-1,2 \mid \pi_{1}^{(2)}, \pi_{2}^{(2)}, \vee\right)$ \\
\hline$W^{\wedge, \vee}$ & $\omega^{\wedge, \vee}:=\left(-1,-1,1,1 \mid \pi_{1}^{(2)}, \pi_{2}^{(2)}, \wedge, \vee\right)$ \\
\hline$W^{\mathrm{mjr}}$ & $\omega^{\mathrm{mjr}}:=\left(-1,-1,-1,3 \mid \pi_{1}^{(3)}, \pi_{2}^{(3)}, \pi_{3}^{(3)}, \mathrm{mjr}\right)$ \\
\hline$W^{\mathrm{mnr}}$ & $\omega^{\mathrm{mnr}}:=\left(-1,-1,-1,3 \mid \pi_{1}^{(3)}, \pi_{2}^{(3)}, \pi_{3}^{(3)}, \mathrm{mnr}\right)$ \\
\hline$W^{\mathrm{mjr}, \mathrm{mnr}}$ & $\omega^{\mathrm{mjr}, \mathrm{mnr}}:=\left(-1,-1,-1,2,1 \mid \pi_{1}^{(3)}, \pi_{2}^{(3)}, \pi_{3}^{(3)}, \mathrm{mjr}, \mathrm{mnr}\right)$ \\
\hline
\end{tabular}

WEIGHTED CLONES FROM WEIGHTINGS IN THEOREM III.1 
$C$ to denote a key weighting of a clone $C$, and the corresponding weighted clone with support $C$ that it generates. Note that a key weighting exists as a weighting of $C$ if, and only if, the operations to which it assigns a positive value are contained in $C$. For example, $\omega_{C}^{\mathbf{0}}$ and $W_{C}^{\mathbf{0}}$ do not exist if $\mathbf{0} \notin C$.

The next result is a simple consequence of Theorem III.1.

Lemma III.2. Let $C$ be a Boolean clone, $\omega_{C}$ be a key weighting of $C$ and let $W_{C}=\left\langle\omega_{C}\right\rangle$. If $\omega_{C}$ is the only key weighting contained in $W_{C}$, then $W_{C}$ is an atom of Lat $(C)$.

By analysing the structure of weightings in the respective weighted clones, we will establish the following results.

Theorem III.3. Let $C$ be a Boolean clone with $\mathbf{0}, \mathbf{1}$ or $\neg \in C$. Then $W_{C}^{0}, W_{C}^{1}$ or $W_{C}^{ᄀ}$ is an atom of Lat $(C)$, respectively.

Theorem III.4. Let $C$ be a Boolean clone. If $\wedge \in C$, then $W_{C}^{\wedge}$ is an atom of if, and only if, $\mathbf{0} \notin C$. Similarly, if $\vee \in C$, $W_{C}^{\vee}$ is an atom of Lat $(C)$ if, and only if, $\mathbf{1} \notin C$.

As our main result, we show the following result.

Theorem III.5. Let $C$ be a Boolean clone with $\wedge, \vee \in C$. Then $W_{C}^{\wedge, \vee}$ is an atom of Lat $(C)$.

The weighted clone $W_{C}^{\wedge, \vee}$ corresponds, via the Galois connection established in [6], to the set of valued constraints defined by submodular functions, which are a fundamental concept in combinatorial optimisation [21]. Hence Theorem III.5 implies that the corresponding valued constraint languages (which are strictly contained in the class of all general-valued submodular functions if $C$ is strictly larger than Clone $(\{\wedge, \vee\}))$ are maximal. This was previously shown by different means for $C=\operatorname{Clone}(\{\wedge, \vee\})$ and $C=\mathbf{O}_{D}$ (where $D$ is any finite set) in [7].

We complete the picture of minimal Boolean weighted clones by establishing the following results.

Theorem III.6. Let $C$ be a Boolean clone. Then $W_{C}^{\mathrm{mjr}}$ is an atom of Lat $(C)$ if, and only if, mjr is the only key operation in $C$, and $W_{C}^{\mathrm{mnr}}$ is an atom of Lat $(C)$ if, and only if, $\mathrm{mnr}$ is the only key operation in $C$.

Theorem III.7. Let $C$ be a Boolean clone with $\mathrm{mjr}, \mathrm{mnr} \in C$. Then $W_{C}^{\mathrm{mjr}, \mathrm{mnr}}$ is an atom of Lat $(C)$ if, and only if $\wedge, \vee \notin C$.

Note that these results are sufficient to establish the minimal weighted clones with support $C$ for every Boolean clone $C$. Some of the proofs we give below also fully characterise the weightings in these minimal weighted clones. Obvious directions for future work are to try to extend these results to non-Boolean domains, and to obtain complete descriptions of all possible weighted clones.

\section{Proof of Theorem III.3}

For any $\alpha \in D$, we define a corresponding unary constant operation $\boldsymbol{\alpha}: D \rightarrow D$, where $\boldsymbol{\alpha}: x \mapsto \alpha$ for all $x \in D$. For any clone $C$ with $\alpha \in C$, we define the weighting $\omega_{C}^{\alpha}:=$ $\left(-1,1 \mid \pi_{1}^{(1)}, \boldsymbol{\alpha}\right)$ and the weighted clone $W_{C}^{\boldsymbol{\alpha}}:=\left\langle\omega_{C}^{\boldsymbol{\alpha}}\right\rangle$.
Proposition IV.1. Let $\alpha \in D$ and $C$ be a clone with $\alpha \in C$. A weighting $\omega$ of $C$ is an element of $W_{C}^{\alpha}$ if, and only if, $\omega$ is of the form

$$
\left(-q_{1}, \ldots,-q_{n}, \sum_{i=1}^{n} q_{i} \mid \pi_{1}^{(k)}, \ldots, \pi_{n}^{(k)}, \boldsymbol{\alpha}\right),
$$

where $q_{i} \geq 0$ for each $i \in\{1, \ldots, n\}$.

Proof. By Lemma II.7, $\omega \in W_{C}^{\alpha}$ if, and only if,

$$
\omega=\sum_{i=1}^{n} c_{i} \cdot \omega_{C}^{\boldsymbol{\alpha}}\left[g_{i}\right]=\sum_{i=1}^{n} c_{i} \cdot\left(-1,1 \mid g_{i}, \boldsymbol{\alpha}\right),
$$

where each $g_{i} \in C^{(k)}$. Since weightings assign negative values only to projections, all of the $g_{i}$ must be $k$-ary projections.

The following proposition shows that the $W_{C}^{\boldsymbol{\alpha}}$ are atoms if $\alpha \in C$, thus establishing the first two cases of Theorem III.3.

Proposition IV.2. Let $C$ be a clone with $\boldsymbol{\alpha} \in C$. Then $W_{C}^{\boldsymbol{\alpha}}$ is an atom of $\operatorname{Lat}(C)$.

Proof. Let $\omega$ be a non-zero $k$-ary weighting in $W_{C}^{\alpha}$. By Proposition IV.1, only $\boldsymbol{\alpha}$ gets positive weight in $\omega$ and we have

$$
\omega[\underbrace{\pi_{1}^{(1)}, \ldots, \pi_{1}^{(1)}}_{k \text { times }}]=\left(-q, q \mid \pi_{1}^{(1)}, \boldsymbol{\alpha}\right),
$$

with $q>0$. Since $\omega\left[\pi_{1}^{(1)}, \ldots, \pi_{1}^{(1)}\right]$ is equal to $q \cdot \omega^{\boldsymbol{\alpha}}$ we have that $\omega$ generates $\omega_{C}^{\boldsymbol{\alpha}}$ and hence $W_{C}^{\boldsymbol{\alpha}}$, so $W_{C}^{\boldsymbol{\alpha}}$ is minimal.

For an operation $g$, we denote by $\neg g$ the operation $\neg[g]$. The following proposition is essentially [25, Theorem 4.1].

Proposition IV.3. . Let $C$ be a clone with $\neg \in C$. A weighting $\omega$ of $C$ is an element of $W_{C}$ if, and only if, $\omega$ is of the form

$$
\left(-q_{1}, \ldots,-q_{k}, q_{1}, \ldots, q_{k} \mid \pi_{1}^{(k)}, \ldots, \pi_{k}^{(k)}, \neg \pi_{1}^{(k)}, \ldots, \neg \pi_{k}^{(k)}\right),
$$

where $q_{i} \geq 0$ for each $i \in\{1, \ldots, k\}$.

Proof. By Lemma II.7, $\omega \in W_{C}^{\neg}$ if, and only if,

$$
\begin{aligned}
\omega & =\sum_{i=1}^{k} c_{i} \cdot \omega_{C}\left[g_{i}\right] \\
& =\sum_{i=1}^{k} c_{i} \cdot\left(-1,1 \mid g_{i}, \neg g_{i}\right),
\end{aligned}
$$

where each $g_{i} \in C^{(k)}$. As $\omega$ can assign negative weight only to projections, the terms in (3) give the required form.

The following proposition shows that $W_{C}$ is an atom if $\neg \in C$, thus establishing the third case of Theorem III.3.

Proposition IV.4. Let $C$ be a clone with $\neg \in C$. Then $W_{C}$ is an atom of $\operatorname{Lat}(C)$.

Proof. Let $\omega$ be a non-zero $k$-ary weighting in $W_{C}^{\neg}$. By Proposition IV.3,

$$
\omega[\underbrace{\pi_{1}^{(1)}, \ldots, \pi_{1}^{(1)}}_{k \text { times }}]=\left(-q, q \mid \pi_{1}^{(1)}, \neg \pi_{1}^{(1)}\right),
$$


where $q>0$. As $\omega\left[\pi_{1}^{(1)}, \ldots, \pi_{1}^{(1)}\right]=q \cdot \omega_{C}^{\neg}$, the weighting $\omega$ generates $\omega_{C}$ and hence $W_{C}^{\neg}$, so $W_{C}$ is minimal.

\section{Proof of Theorem III.4}

We now give a description of the weightings in the weighted clones $W_{C}^{\wedge}$ and $W_{C}^{\vee}$ and use it to prove Theorem III.4. A description of weightings in $W_{\Lambda \mathrm{P}}^{\wedge}$, where $\Lambda \mathrm{P}=$ Clone $(\{\Lambda\})$ was obtained in [25] using Farkas lemma. For our more general treatment of $W_{C}^{\wedge}$ and $W_{C}^{\vee}$, we use the notion of dependence [24], which is motivated by the following observation.

Lemma V.1. Suppose that $f_{1}, f_{2} \in \mathbf{O}_{D}^{(k)}$ are operations and $\alpha, \beta \in D$ such that for any $\mathrm{x} \in D^{k}$ we have

$$
f_{1}(\mathbf{x})=\alpha \Longrightarrow f_{2}(\mathbf{x})=\beta \text {. }
$$

Then for any $g_{1}, \ldots, g_{k} \in \mathbf{O}_{D}^{(\ell)}$ and $\mathbf{y} \in D^{\ell}$, we have

$$
f_{1}\left[g_{1}, \ldots, g_{k}\right](\mathbf{y})=\alpha \Longrightarrow f_{2}\left[g_{1}, \ldots, g_{k}\right](\mathbf{y})=\beta \text {. }
$$

Definition V.2. If $f_{1}, f_{2} \in \mathbf{O}_{D}^{(k)}$ are distinct operations and $\alpha, \beta \in D$ such that, for all $\mathbf{x} \in D^{k}$, we have

$$
f_{1}(\mathbf{x})=\alpha \Longrightarrow f_{2}(\mathbf{x})=\beta,
$$

then we say that $f_{2}$ is $(\boldsymbol{\alpha} \rightarrow \boldsymbol{\beta})$-dependent on $f_{1}$.

Moreover, we say that $f \in \mathbf{O}_{D}^{(k)}$ is $(\boldsymbol{\alpha} \rightarrow \boldsymbol{\beta})$-dependent on $T \subseteq\{1, \ldots, k\}$ if there is $i \in T$ such that $f$ is $(\boldsymbol{\alpha} \rightarrow \boldsymbol{\beta})$ dependent on $\pi_{i}^{(k)}$ and $f$ is not a projection.

We now give a characterisation of the weightings in $W_{C}^{\wedge}$. Note that, for any $k$-ary non-projection operation $f$, the operation $\pi_{i}^{(k)} \wedge f$ is $(0 \rightarrow 0)$-dependent on $\pi_{i}^{(k)}$. This is a crucial observation for the description of $W_{C}^{\wedge}$.

Proposition V.3. Let $C$ be a clone with $\wedge \in C$. A k-ary weighting $\omega$ of $C$ is an element of the weighted clone $W_{C}^{\wedge}$ if, and only if,

- $\omega$ assigns positive weights only to non-projections that are $(0 \rightarrow 0)$-dependent on some $k$-ary projection;

- for every subset $T \subseteq\{1, \ldots, k\}$,

$$
-\sum_{i \in T} \omega\left(\pi_{i}^{(k)}\right) \leq \sum_{\substack{f \in C^{(k)} \\(0 \rightarrow 0) \text {-dependent on } T}} \omega(f) .
$$

Proof. First, suppose that $\omega \in W_{C}^{\wedge}$. We define

$$
\omega_{C}^{\wedge^{01}}:=\omega_{C}^{\wedge}\left[\pi_{1}^{(2)}, \wedge\right]=\left(-1,1 \mid \pi_{1}^{(2)}, \wedge\right) .
$$

We have $\omega_{C}^{\wedge 01}\left[\pi_{1}^{(2)}, \pi_{2}^{(2)}\right]+\omega_{C}^{\wedge 01}\left[\pi_{2}^{(2)}, \pi_{1}^{(2)}\right]=\omega^{\wedge}$, and thus

$$
\left\langle\omega_{C}^{\wedge}\right\rangle=\left\langle\omega_{C}^{\wedge} \stackrel{10}{1}^{\wedge}=W_{C}^{\wedge}\right.
$$

Hence, by Lemma II.7, there are $k$-ary operations $g_{1}^{j}, g_{2}^{j} \in$ $C$ and constants $c_{j} \in \mathbb{Q}_{\geq 0}$ such that

$$
\begin{aligned}
\omega & =\sum_{j=1}^{n} c_{j} \cdot \omega_{C}^{\wedge^{01}}\left[g_{1}^{j}, g_{2}^{j}\right] \\
& =\sum_{j=1}^{n} c_{j} \cdot\left(-1,1 \mid g_{1}^{j}, g_{1}^{j} \wedge g_{2}^{j}\right) .
\end{aligned}
$$

We will now show that there is a subset $T \subseteq\{1, \ldots, k\}$ and for each $i \in T$ an indexing set $T_{i}$ such that

$$
\omega=\sum_{i \in T} \sum_{j \in T_{i}} c_{i j} \cdot\left(-1,1 \mid \pi_{i}^{(k)}, \pi_{i}^{(k)} \wedge f_{j}^{i}\right),
$$

for some $f_{j}^{i} \in C$, which proves that $\omega$ is of the required form.

First note that we can rewrite (5) as

$$
\omega=\sum_{i \in S} \sum_{j \in S_{i}} c_{i j}^{\prime} \cdot\left(-1,1 \mid g_{i}, g_{i} \wedge h_{j}^{i}\right),
$$

where $g_{i}, h_{j}^{i} \in C$ with $g_{i}$ distinct, and $S$ and $S_{i}$ are indexing sets.

If for each $i \in S$ the operation $g_{i}$ is a projection, then the expression in (7) is of the form (6) and we are done. Otherwise there is some $m \in S$ such that $g_{m}$ is a non-projection. In this case the sum of the positive and negative weights assigned to $g_{m}$ by the terms in (7) has to be non-negative, as otherwise $\omega\left(g_{m}\right)<0$, so $\omega$ would not be a valid weighting.

We can cancel out positive and negative weights assigned to $g_{m}$ and preserve the form of expression (7). Suppose $g_{\ell}$ and $h_{r}^{\ell}$ are such that $g_{m}=g_{\ell} \wedge h_{r}^{\ell}$. Note that we can find an operation $h_{s}^{m}$ with $s \in S_{m}$ such that $c_{m s}^{\prime} \leq c_{\ell r}^{\prime}$, if necessary we split the weighting with coefficient $c_{m s}^{\prime}$ into two. We replace the weightings $c_{\ell r}^{\prime} \cdot\left(-1,1 \mid g_{\ell}, g_{\ell} \wedge h_{r}^{\ell}\right)$ and $c_{m s}^{\prime} \cdot\left(-1,1 \mid g_{m}, g_{m} \wedge h_{s}^{m}\right)$ by

$$
\left(c_{\ell r}^{\prime}-c_{m s}^{\prime}\right) \cdot\left(-1,1 \mid g_{\ell}, g_{\ell} \wedge h_{r}^{\ell}\right)
$$

and

$$
c_{m s}^{\prime} \cdot\left(-1,1 \mid g_{\ell}, g_{\ell} \wedge\left(h_{r}^{\ell} \wedge h_{s}^{m}\right)\right) .
$$

After identifying all operations assigned non-negative weights and performing all possible eliminations of this kind, we obtain a weighting of the form (6).

For the other direction suppose that $\omega \in \mathbf{W}_{C}^{(k)}$ satisfies the stated conditions. To show that $\omega$ is in the weighted clone $W_{C}^{\wedge}$ we shall show that $\omega$ corresponds to a flow in a certain network, and all such flows correspond to elements of $W_{C}^{\wedge}$.

For any $k \geq 2$, we construct a directed network $G_{k}$, with vertices $V_{1} \cup V_{2} \cup\{s, t\}$, where $V_{1}$ is the set of all $k$-ary projections on $B, V_{2}$ is the set of all $k$-ary non-projections on $B$ that are $(0 \rightarrow 0)$-dependent on some projection, and $s$ and $t$ are two additional source and sink vertices. The edges of $G_{k}$ are all possible edges of the following three types: either $\left(s, \pi_{i}^{(k)}\right)$, where $\pi_{i}^{(k)} \in V_{1}$, or $\left(\pi_{i}^{(k)}, f\right)$, where $\pi_{i}^{(k)} \in V_{1}$ and $f \in V_{2}$ is $(0 \rightarrow 0)$-dependent on $\pi_{i}^{(k)}$, or $(f, t)$, where $f \in V_{2}$.

The network $G_{2}$ is shown in Figure 1, where the binary operations are defined in Table II.

We set the capacity on each edge into $\pi_{i}^{(k)}$ to be $-\omega\left(\pi_{i}^{(k)}\right)$, which is nonnegative by the first inequality in (4), the capacity on each edge out of $f \in V_{2}$ to be $\omega(f)$, and all other edge capacities to be infinite. Then by the second inequality in (4), the minimum cut in the network has capacity $-\sum_{i=1}^{k} \omega\left(\pi_{i}^{(k)}\right)=\sum_{f \in V_{2}} \omega(f)$. Hence, by the max-flow min-cut theorem there is a flow where each edge out of $s$ and each edge into $t$ carries its maximum capacity. 
TABLE II

BINARY OPERATIONS $(0 \rightarrow 0)$-DEPENDENT ON SOME $\pi_{i}^{(2)}$

\begin{tabular}{|c||c|c|c|c|}
\hline input & $\wedge$ & $\mathrm{op}_{1}$ & $\mathrm{op}_{2}$ & $\mathbf{0}$ \\
\hline$(0,0)$ & 0 & 0 & 0 & 0 \\
$(0,1)$ & 0 & 1 & 0 & 0 \\
$(1,0)$ & 0 & 0 & 1 & 0 \\
$(1,1)$ & 1 & 0 & 0 & 0 \\
\hline
\end{tabular}

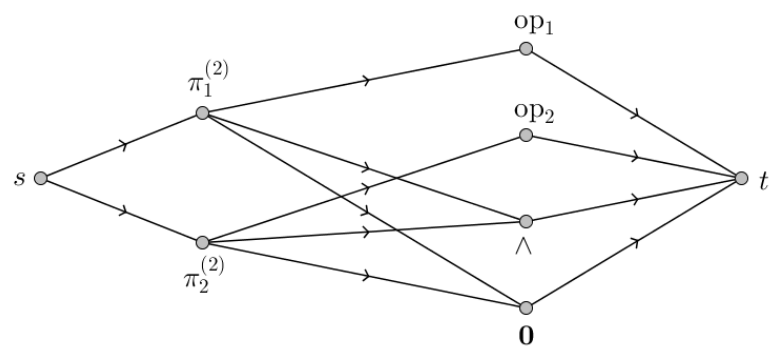

Fig. 1. Directed network $G_{2}$

Finally, we note that any valid flow on $G_{k}$ is determined by the flows along the edges of the form $\left(\pi_{i}^{(k)}, f\right)$, where $\pi_{i}^{(k)} \in V_{1}$ and $f \in V_{2}$. Hence any flow in $G_{k}$ can be associated with a weighting in $W_{C}^{\wedge}$ by adding nonnegative scalings of weightings of the form $\left(-1,1 \mid \pi_{i}^{(k)}, f\right)=\omega_{C}^{\wedge 1}\left[\pi_{i}^{(k)}, f\right]$, where each nonnegative scaling factor is given by the flow on the edge $\left(\pi_{i}^{(k)}, f\right)$.

We note that, dually, a similar description holds for the weighted clone $W_{C}^{\vee}$ with $(0 \rightarrow 0)$-dependence replaced by $(1 \rightarrow 1)$-dependence. A similar proof technique was used in [8].

The corollary below follows from Proposition V.3 and Proposition IV.1.

\section{Corollary V.4. Let $C$ be a clone. We have that}

1) $W_{C}^{\mathbf{0}} \subsetneq W_{C}^{\wedge}$ if the support clone $C$ contains $\mathbf{0}$ and $\wedge$, and

2) $W_{C}^{1} \subsetneq W_{C}^{\vee}$ if the support clone $C$ contains 1 and $\vee$.

Finally, we prove Thorem III.4, which tells us that $W_{C}^{\wedge}$ is an atom of Lat $(C)$ if, and only if, $\wedge \in C$ and $\mathbf{0} \notin C$.

Proof of Theorem III.4. First we show that the condition is necessary for $W_{C}^{\wedge}$ to be an atom. If $\wedge \notin C$, then $W_{C}^{\wedge}$ would not exist. If $\mathbf{0} \in C$ then $W_{C}^{\mathbf{0}} \in \mathrm{Lat}(C)$ and, by Corollary V.4, $W_{C}^{0} \subsetneq W_{C}^{\wedge}$, which means that $W_{C}^{\wedge}$ is not an atom.

Now we show that the condition is sufficient. The only key operations that are $(0 \rightarrow 0)$-dependent on projections are $\mathbf{0}$ and $\wedge$. Thus, by Proposition V.3, the only key weightings that might belong to $W_{C}^{\wedge}$ are $\omega_{C}^{\mathbf{0}}$ and $\omega_{C}^{\wedge}$. Since $\mathbf{0} \notin C, \omega_{C}^{\mathbf{0}}$ is not a weighting of $C$. Therefore, $\omega_{C}^{\wedge}$ is the only key weighting in $W_{C}^{\wedge}$, and so by Lemma III. $2 W_{C}^{\wedge}$ is an atom of Lat $(C)$.

An analogous argument gives the second case of Theorem III.4.

\section{Proof of Theorem III.5}

The following proposition gives a property that all weightings in $W_{C}^{\wedge, \vee}$ satisfy, and this will be sufficient to show that $W_{C}^{\wedge}, \vee$ is an atom.
Proposition VI.1. Let $C$ be a clone containing $\wedge$ and $\vee$, and let $\omega \in W_{C}^{\wedge, \vee}$ be a non-zero $k$-ary weighting of $C$. Then for every projection $\pi_{i}^{(k)}$ with $\omega\left(\pi_{i}^{(k)}\right)<0$, there are operations $g, h \in C^{(k)}$ with $\omega(g), \omega(h)>0$ such that $g$ is $(0 \rightarrow 0)$-dependent on $\pi_{i}^{(k)}$ and $h$ is $(1 \rightarrow 1)$-dependent on $\pi_{i}^{(k)}$.

Proof. Let $\pi_{i}^{(k)}$ be a projection with $\omega\left(\pi_{i}^{(k)}\right)<0$. Note that such a projection exists as $\omega$ is non-zero and assigns negative weights only to projections. We shall show that there exists an operation $g$ with $\omega(g)>0$ that is $(0 \rightarrow 0)$-dependent on $\pi_{i}^{(k)}$. We will find such a function by iteration.

By Lemma II.7, for some $g_{i, 1}, g_{i, 2} \in C^{(k)}$,

$$
\omega=\sum_{i=1}^{m} c_{i} \cdot \omega_{C}^{\wedge, \vee}\left[g_{i, 1}, g_{i, 2}\right] .
$$

As $\omega\left(\pi_{i}^{(k)}\right)<0$, a non-zero term $\omega_{C}^{\wedge, \vee}\left[\pi_{i}^{(k)}, g_{0}^{\prime}\right]$ appears in the summation (8) for some $g_{0}^{\prime} \in C^{(k)}$. Let $g_{1}=\pi_{i}^{(k)} \wedge g_{0}^{\prime}$. If $\omega\left(g_{1}\right)>0$, as $g_{1}$ is $(0 \rightarrow 0)$-dependent on $\pi_{i}^{(k)}$, then we have found a suitable operation $g$. Otherwise, $\omega\left(g_{1}\right)=0$ and so there is an operation $g_{1}^{\prime} \in C^{(k)}$ such that a non-zero term $\omega_{C}^{\wedge}, \vee\left[g_{1}, g_{1}^{\prime}\right]$ appears in the summation (8). For $i>1$ we define $g_{i}=g_{i-1} \wedge g_{i-1}^{\prime}$. If $\omega\left(g_{i}\right)>0$, as the operation $g_{i}$ is $(0 \rightarrow 0)$ dependent on $g_{i-1}$ and so on $\pi_{i}^{(k)}$, then we have found a suitable operation $g$. Otherwise, $\omega\left(g_{i}\right)=0$ and there is an operation $g_{i}^{\prime}$ such that a non-zero term $\omega\left[g_{i}, g_{i}^{\prime}\right]$ appears in the summation (8). We repeat this process until we find an operation $g_{i}$ with $\omega\left(g_{i}\right)>0$.

Note that for each $i \geq 0$ we have that $g_{i+1}$ is $(0 \rightarrow 0)$ dependent on $g_{i}$ and hence $g_{i}$ assigns strictly more zeros than $g_{i+1}$ to tuples in $D^{k}$. The above process must terminate as $C^{(k)}$ is finite and no two operations among $g_{i}$ can be equal.

Similarly, we can show the existence of an operation $h$ with $\omega(h)>0$ that is $(1 \rightarrow 1)$-dependent on $\pi_{i}^{(k)}$.

Proof of Theorem III.5. First note that the condition is necessary for $W_{C}^{\wedge, \vee}$ to be an atom. If $\wedge$ or $\vee$ is not in $C$, then $W_{C}^{\wedge, \vee}$ does not exist and so $W_{C}^{\wedge, \vee}$ is not an atom of Lat $(C)$.

Now we show that the condition is sufficient. If $\wedge$ and $\vee$ are in $C$, then $\omega_{C}^{\wedge, \vee}$ is a weighting of $C$. We claim that every other possible key weighting violates properties listed in Proposition VI.1 and so $\omega_{C}^{\wedge, \vee}$ is the only key weighting in $W_{C}^{\wedge, \vee}$. Hence, by Lemma III.2 $W_{C}^{\wedge, \vee}$ is an atom of Lat $(C)$.

To establish this claim note that the key weighting $\omega_{C}^{\neg} \notin$ $W_{C}^{\wedge, \vee}$ as $\neg$ is neither $(0 \rightarrow 0)$ nor $(1 \rightarrow 1)$-dependent on $\pi_{1}^{(1)}$. The key weightings $\omega_{C}^{\mathbf{0}} \notin W_{C}^{\wedge, \vee}$ and $\omega_{C}^{1} \notin W_{C}^{\wedge, \vee}$ as these weightings do not assign a positive weight to any $(1 \rightarrow 1)$ and $(0 \rightarrow 0)$-dependent operation, respectively. The key weightings $\omega_{C}^{\wedge} \notin W_{C}^{\wedge, \vee}$ and $\omega_{C}^{\vee} \notin W_{C}^{\wedge, \vee}$ as these weightings do not assign a positive weight to any $(1 \rightarrow 1)$ and $(0 \rightarrow 0)$-dependent operation, respectively. The key weightings $\omega_{C}^{\mathrm{mjr}}, \omega_{C}^{\mathrm{mnr}}$, and $\omega_{C}^{\mathrm{mjr}, \mathrm{mnr}} \notin W_{C}^{\wedge, \vee}$ as neither mjr nor $\mathrm{mnr}$ is $(0 \rightarrow 0)$ or $(1 \rightarrow 1)$ dependent on any ternary projection.

\section{PRoOf OF THEOREM III.6}

First we show that weighted clones $W^{\mathrm{mjr}}, W^{\mathrm{mnr}}$ are always equal to the top element of the lattice of weighted clones. 
Proposition VII.1. Let $C$ be a clone. If $C$ contains mjr, then $W_{C}^{\mathrm{mjr}}=\mathbf{W}_{C}$. If $C$ contains $\mathrm{mnr}$, then $W_{C}^{\mathrm{mnr}}=\mathbf{W}_{C}$.

Proof. Note that

$$
\omega_{C}^{\mathrm{mjr}}\left[\pi_{1}^{(2)}, \pi_{2}^{(2)}, \pi_{2}^{(2)}\right]=\left(-1,1 \mid \pi_{1}^{(2)}, \pi_{2}^{(2)}\right)
$$

and

$$
\omega_{C}^{\mathrm{mnr}}\left[\pi_{1}^{(2)}, \pi_{1}^{(2)}, \pi_{2}^{(2)}\right]=\left(-1,1 \mid \pi_{1}^{(2)}, \pi_{2}^{(2)}\right) .
$$

Both of these weightings assign a positive weight to a projection operation. So by Lemma II.8, we have that $\omega_{C}^{\mathrm{mjr}}$ and $\omega_{C}^{\mathrm{mnr}}$, when they exist, generate $\mathbf{W}_{C}$.

Proof of Theorem III.6. First we show that the condition is necessary for $W_{C}^{\mathrm{mjr}}$ to be an atom. Suppose, that mjr is not the only key operation in $C$. First, note that mjr $\in C$ as otherwise $W_{C}^{\mathrm{mjr}}$ would not exist. If $C$ also contains a key operation $f$ equal to one of $\mathbf{0}, \mathbf{1}, \neg, \wedge$ or $\vee$, then we have that $W_{C}^{f} \neq \mathbf{W}_{C}$, by our earlier results, and so $W_{C}^{f} \subsetneq W_{C}^{\mathrm{mjr}}$ by Proposition VII.1. If $f=\mathrm{mnr}$, then as $W_{C}^{\mathrm{mjr}}=\mathbf{W}_{C}$ we have that $\omega_{C}^{\mathrm{mjr}, \mathrm{mnr}} \in W_{C}^{\mathrm{mjr}}$, but $W_{C}^{\mathrm{mir}, \mathrm{mnr}} \neq \mathbf{W}_{C}$ by the proof of Theorem III.7, below. Hence in all cases $W_{C}^{\mathrm{mjr}}$ is not an atom.

Now we show that the condition is sufficient. Suppose that mjr is the only key operation in $C$. Then $\omega_{C}^{\mathrm{mjr}}$ is the only key weighting in $W_{C}^{\mathrm{mjr}}$ and so by Lemma III.2 we have that $W_{C}^{\mathrm{mjr}}$ is an atom of Lat $(C)$.

An analogous argument establishes the result for $W_{C}^{\mathrm{mnr}}$.

\section{Proof of TheOREM III.7}

We prove the following lemma that is needed to give the conditions for $W^{\mathrm{mjr}, \mathrm{mnr}}$ to be an atom.

Lemma VIII.1. Let $C$ be a clone that contains $\wedge, \vee, \mathrm{mjr}$ and mnr. Then $W_{C}^{\wedge, \vee} \subsetneq W_{C}^{\mathrm{mjr}, \mathrm{mnr}}$.

Proof. We have that $W_{C}^{\wedge, \vee} \neq W_{C}^{\mathrm{mjr}, \mathrm{mnr}}$ as $\omega_{C}^{\mathrm{mjr}, \mathrm{mnr}} \notin W_{C}^{\wedge, \vee}$ by Proposition VI.1. Also, note that $\omega_{C}^{\mathrm{mjr}, \mathrm{mnr}}\left[\pi_{1}^{(2)}, \pi_{2}^{(2)}, \mathrm{V}\right]=$ $\left(-1,-1,1,1 \mid \pi_{1}^{(2)}, \pi_{2}^{(2)}, \wedge, \vee\right)=\omega_{C}^{\wedge, \vee}$. Hence $\omega_{C}^{\wedge, \vee} \in$ $\left\langle\omega_{C}^{\mathrm{mjr}, \mathrm{mnr}}\right\rangle$ and so $W_{C}^{\wedge, \vee} \subseteq W_{C}^{\mathrm{mjr}, \mathrm{mnr}}$.

Proof of Theorem III.7. First note that by Lemma VIII.1 the condition is necessary for $W_{C}^{\mathrm{mjr}, \mathrm{mnr}}$ to be an atom of Lat $(C)$.

Now we show that the condition is sufficient. We will show that if mjr, mnr $\in C$ and $\wedge, \vee \notin C$, then $W_{C}^{\mathrm{mjr}, \mathrm{mnr}}$ contains no other key weightings apart from $\omega_{C}^{\mathrm{mir}, \mathrm{mnr}}$, and so is an atom of Lat $(C)$ by Lemma III.2. Note that only two non-zero unary weighted maps (not weightings) may be obtained by superposition from $\omega_{C}^{\mathrm{mir}, \mathrm{mnr}}$; these are $\left(-1,1,1,-1 \mid \pi_{1}^{(1)}, \mathbf{0}, \mathbf{1}, \neg\right)$, and $\left(1,-1,-1,1 \mid \pi_{1}^{(1)}, \mathbf{0}, \mathbf{1}, \neg\right)$. Therefore by Lemma II.7 $W_{C}^{\mathrm{mjr}, \mathrm{mnn}}$ contains no unary weightings. So $\omega_{C}^{0}, \omega_{C}^{1}$ and $\omega_{C}^{\neg}$ are never contained in $W_{C}^{\mathrm{mjr}, \mathrm{mnr}}$. This also implies that $W_{C}^{\mathrm{mjr}, \mathrm{mnr}} \neq$ $\mathbf{W}_{C}$. Therefore $\omega_{C}^{\mathrm{mjr}}$ and $\omega_{C}^{\mathrm{mnr}}$ are not contained in $W_{C}^{\mathrm{mjr}, \mathrm{mnr}}$.

Note that if $C$ contains mjr, mnr, and at least one of $\wedge$ and $\vee$, then $C$ contains all four operations. In such a case, $W_{C}^{\wedge, \vee} \subsetneq W_{C}^{\mathrm{mjr}, \mathrm{mnr}}$, by Lemma VIII.1, and so $W_{C}^{\mathrm{mjr}, \mathrm{mnr}}$ is not an atom. Hence, $\omega_{C}^{\wedge}$ and $\omega_{C}^{\vee}$ are not contained in $W_{C}^{\mathrm{mjr} r \mathrm{mnr}}$.

\section{REFERENCES}

[1] L. Barto and M. Kozik, "Constraint Satisfaction Problems Solvable by Local Consistency Methods," Journal of the ACM, vol. 61, no. 1, 2014, article No. 3.

[2] L. Barto, M. Kozik, and T. Niven, "The CSP dichotomy holds for digraphs with no sources and no sinks (a positive answer to a conjecture of Bang-Jensen and Hell)," SIAM Journal on Computing, vol. 38, no. 5, pp. 1782-1802, 2009.

[3] A. Bulatov, "A dichotomy theorem for constraint satisfaction problems on a 3-element set," Journal of the ACM, vol. 53, no. 1, pp. 66-120, 2006.

[4] A. Bulatov, A. Krokhin, and P. Jeavons, "Classifying the Complexity of Constraints using Finite Algebras," SIAM Journal on Computing, vol. 34, no. 3, pp. 720-742, 2005.

[5] A. A. Bulatov, "Complexity of conservative constraint satisfaction problems," ACM Transactions on Computational Logic, vol. 12, no. 4, 2011, article 24.

[6] D. A. Cohen, M. C. Cooper, P. Creed, P. Jeavons, and S. Živný, "An algebraic theory of complexity for discrete optimisation," SIAM Journal on Computing, vol. 42, no. 5, pp. 915-1939, 2013.

[7] D. A. Cohen, M. C. Cooper, P. G. Jeavons, and A. A. Krokhin, "The Complexity of Soft Constraint Satisfaction," Artificial Intelligence, vol. 170, no. 11, pp. 983-1016, 2006.

[8] D. A. Cohen, P. G. Jeavons, and S. Živný, "The expressive power of valued constraints: Hierarchies and collapses," Theoretical Computer Science, vol. 409, no. 1, pp. 137-153, 2008.

[9] R. Dechter, Constraint Processing. Morgan Kaufmann, 2003.

[10] T. Feder and M. Y. Vardi, "The Computational Structure of Monotone Monadic SNP and Constraint Satisfaction: A Study through Datalog and Group Theory," SIAM J. on Computing, vol. 28, no. 1, pp. 57-104, 1998.

[11] P. Hell and J. Nešetřil, "Colouring, constraint satisfaction, and complexity," Computer Science Review, vol. 2, no. 3, pp. 143-163, 2008.

[12] A. Huber, A. Krokhin, and R. Powell, "Skew bisubmodularity and valued CSPs," SIAM J. on Computing, vol. 43, no. 3, pp. 1064-1084, 2014.

[13] P. M. Idziak, P. Markovic, R. McKenzie, M. Valeriote, and R. Willard, "Tractability and learnability arising from algebras with few subpowers," SIAM Journal on Computing, vol. 39, no. 7, pp. 3023-3037, 2010.

[14] P. Jeavons, A. Krokhin, and S. Živný, "The complexity of valued constraint satisfaction," Bulletin of the European Association for Theoretical Computer Science (EATCS), vol. 113, pp. 21-55, 2014.

[15] P. G. Jeavons, "On the Algebraic Structure of Combinatorial Problems," Theoretical Computer Science, vol. 200, no. 1-2, pp. 185-204, 1998.

[16] P. G. Jeavons, D. A. Cohen, and M. Gyssens, "Closure Properties of Constraints," Journal of the ACM, vol. 44, no. 4, pp. 527-548, 1997.

[17] P. G. Kolaitis and M. Y. Vardi, "Conjunctive-Query Containment and Constraint Satisfaction," Journal of Computer and System Sciences, vol. 61, no. 2, pp. 302-332, 2000.

[18] V. Kolmogorov, A. A. Krokhin, and M. Rolinek, "The complexity of general-valued CSPs," in 56th Annual IEEE Symposium on Foundations of Computer Science, FOCS, 2015, pp. 1246-1258.

[19] V. Kolmogorov and S. Živný, "The complexity of conservative valued CSPs," Journal of the ACM, vol. 60, no. 2, 2013, article No. 10.

[20] M. Kozik and J. Ochremiak, "Algebraic properties of valued constraint satisfaction problem," in Proc. of the 42nd International Colloquium on Automata, Languages and Programming (ICALP'15), ser. Lect. Notes in Computer Science, vol. 9134. Springer, 2015, pp. 846-858.

[21] A. Schrijver, Combinatorial Optimization: Polyhedra and Efficiency, ser. Algorithms and Combinatorics. Springer, 2003, vol. 24.

[22] J. Thapper and S. Živný, "The complexity of finite-valued CSPs," Journal of the ACM, to appear.

[23] — , "Necessary Conditions on Tractability of Valued Constraint Languages," SIAM Journal on Discrete Mathematics, vol. 29, no. 4, pp. 2361-2384, 2015.

[24] A. Vaicenavičius, "A study of weighted clones," Master's thesis, Mathematical Institute, University of Oxford, 2014.

[25] J. Vančura, "Weighted Clones," Master's thesis, Department of Algebra, Charles University, 2014.

[26] S. Živný, The complexity of valued constraint satisfaction problems, ser. Cognitive Technologies. Springer, 2012. 